\title{
Peptide synthesis under the alkaline hydrothermal conditions on Enceladus
}

Wataru Takahagi*, Kaito Seo, Takazo Shibuya, Yoshinori Takano, Kosuke Fujishima, Masafumi Saitoh, Shigeru Shimamura, Yohei Matsui, Masaru Tomita and Ken Takai

*Corresponding author: Wataru Takahagi (watarut@eqchem.s.u-tokyo.ac.jp)

\section{This PDF file includes:}

Table S1. Sampling conditions of hydrothermal experiment.

Table S2. Initial condition of Enceladus hydrothermal experiment.

Table S3. Concentration of amino acids under with \& without amino acids experiments

Figure S1. Representative single mass chromatogram of dipeptides.

Figure S2a. Time series patterns showing the formation of dipeptides (Ala- group).

Figure S2b. Time series patterns showing the formation of dipeptides (Asp- group).

Figure S2c. Time series patterns showing the formation of dipeptides (Glu- group).

Figure S2d. Time series patterns showing the formation of dipeptides (Gly-group).

Figure S2e. Time series patterns showing the formation of dipeptides (Ser- group).

Figure S2f. Time series patterns showing the formation of dipeptides (Val-group). 
Manuscript for ACS Earth and Space Chem.

Supplementary Information

\section{Supplementary Information}

Table S1. Initial condition of Enceladus hydrothermal environment

\begin{tabular}{lllll}
\hline & & Value & Unit & Reference \\
\hline \multirow{2}{*}{ Chondrite (Enceladus Core) } & Olivine & 7.57 & $\mathrm{~g}$ & Hsu et al., 2015 \\
& Orthopyroxene & 12.43 & $\mathrm{~g}$ & Hsu et al ., 2015 \\
\hline \multirow{4}{*}{ Amino Acids } & Glycine & 10.00 & $\mathrm{mM}$ & $*$ \\
& L-Alanine & 3.07 & $\mathrm{mM}$ & $*$ \\
& L-Glutamic Acid & 1.01 & $\mathrm{mM}$ & $*$ \\
& L-Valine & 0.81 & $\mathrm{mM}$ & $*$ \\
& L-Serine & 0.27 & $\mathrm{mM}$ & $*$ \\
& L-Aspartic Acid & 0.35 & $\mathrm{mM}$ & $*$ \\
\hline \multirow{3}{*}{ Solutions } & $\mathrm{NaCl}$ & 0.10 & $\mathrm{M}$ & Postberg et al., 2009 \\
& $\mathrm{NaHCO}_{3}$ & 0.05 & $\mathrm{M}$ & Postberg et al., 2009 \\
& $\mathrm{NH}_{3}$ & 1.00 & $\mathrm{M}$ & Sekine et al., 2015 \\
\hline
\end{tabular}

*These amino acid concentration ratio were estimated on the basis of amino acids average included in mateorites collieded on the Earth (Glavin et al., 2010, Pizzarello et al., 2012, Parker et al., 2011, Johnson et al., 2008) 
Manuscript for ACS Earth and Space Chem.

Supplementary Information

Table S2. Sampling conditions of hydrothermal experiment.

\begin{tabular}{|c|c|c|c|c|}
\hline Experiment & Day & Pressure (bar) & Temperature $(\square)$ & Operation \\
\hline \multirow{9}{*}{$\begin{array}{l}\text { With } \\
\text { Amino Acid } \\
\text { and Rocks }\end{array}$} & 0 (October 19,2015$)$ & 1 & 25 (Room Temp.) & up to $100 \square$ \\
\hline & 3 (October 22, 2015) & 200 & 100 & down to $30 \square$ \\
\hline & 7 (October 26, 2015) & 200 & 30 & up to $100 \square$ \\
\hline & 17 (November 5, 2015) & 200 & 100 & down to $30 \square$ \\
\hline & 28 (November 16, 2015) & 200 & 30 & up to $100 \square$ \\
\hline & 52 (December 10, 2015) & 200 & 100 & down to $30 \square$ \\
\hline & 77 (January 4, 2016) & 200 & 30 & up to $100 \square$ \\
\hline & 112 (February 8, 2016) & 200 & 100 & down to $30 \square$ \\
\hline & 147 (March 14, 2016) & 200 & 30 & - \\
\hline \multirow{9}{*}{$\begin{array}{l}\text { Without } \\
\text { Amino Acids }\end{array}$} & 0 (June 13, 2016) & 1 & 25 (Room Temp.) & up to $100 \square$ \\
\hline & 3 (June 16, 2016) & 200 & 100 & down to $30 \square$ \\
\hline & 7 (June 20, 2016) & 200 & 30 & up to $100 \square$ \\
\hline & 17 (June 30, 2016) & 200 & 100 & down to $30 \square$ \\
\hline & 28 (July 11, 2016) & 200 & 30 & up to $100 \square$ \\
\hline & 59 (August 11, 2016) & 200 & 100 & down to $30 \square$ \\
\hline & 94 (September 15, 2016) & 200 & 30 & up to $100 \square$ \\
\hline & 129 (Ocrober 20, 2016) & 200 & 100 & down to $30 \square$ \\
\hline & 164 (November 24, 2016) & 200 & 30 & - \\
\hline \multirow{9}{*}{$\begin{array}{l}\text { Without } \\
\text { Rocks }\end{array}$} & 0 (June 13, 2016) & 1 & 25 (Room Temp.) & up to $100 \square$ \\
\hline & 3 (June 16, 2016) & 200 & 100 & down to $30 \square$ \\
\hline & 7 (June 20, 2016) & 200 & 30 & up to $100 \square$ \\
\hline & 17 (June 30, 2016) & 200 & 100 & down to $30 \square$ \\
\hline & 28 (July 11, 2016) & 200 & 30 & up to $100 \square$ \\
\hline & 52 (August 4, 2016) & 200 & 100 & down to $30 \square$ \\
\hline & 77 (August 29, 2016) & 200 & 30 & up to $100 \square$ \\
\hline & 112 (October 3, 2016) & 200 & 100 & down to $30 \square$ \\
\hline & 147 (November 7, 2016) & 200 & 30 & - \\
\hline
\end{tabular}


Manuscript for ACS Earth and Space Chem.

Supplementary Information

Table S3. Concentration of amino acids under with \& without amino acids experiment.

\begin{tabular}{|c|c|c|c|c|c|c|c|}
\hline \multirow{2}{*}{$\begin{array}{l}\text { Experiment } \\
\text { Unit }\end{array}$} & \multirow[t]{2}{*}{ Day } & Alanine & \multicolumn{3}{|c|}{ Aspartate Glutamate Glycine } & \multirow[t]{2}{*}{ Serine } & \multirow[t]{2}{*}{ Valine } \\
\hline & & & & & $\mathrm{M}$ & & \\
\hline \multirow{9}{*}{$\begin{array}{l}\text { With } \\
\text { Amino Acid }\end{array}$} & 0 & 2.199 & 0.392 & 0.452 & 11.339 & 0.211 & 0.864 \\
\hline & 3 & 1.781 & 0.319 & 0.050 & 7.319 & 0.092 & 0.874 \\
\hline & 7 & 1.101 & 0.166 & 0.022 & 3.030 & 0.050 & 0.432 \\
\hline & 17 & 0.805 & 0.132 & 0.015 & 1.903 & 0.037 & 0.322 \\
\hline & 28 & 2.326 & 0.396 & 0.055 & 11.742 & 0.127 & 1.116 \\
\hline & 52 & 2.308 & 0.495 & 0.069 & 12.289 & 0.113 & 1.180 \\
\hline & 77 & 2.358 & 0.498 & 0.072 & 11.176 & 0.131 & 1.029 \\
\hline & 112 & 2.845 & 0.504 & 0.068 & 6.818 & 0.077 & 1.333 \\
\hline & 147 & 2.347 & 0.419 & 0.072 & 7.901 & 0.108 & 1.213 \\
\hline \multirow{9}{*}{$\begin{array}{l}\text { Without } \\
\text { Amino Acids }\end{array}$} & 0 & n.d. & n.d. & n.d. & n.d. & n.d. & n.d. \\
\hline & 3 & n.d. & n.d. & n.d. & n.d. & n.d. & n.d. \\
\hline & 7 & n.d. & n.d. & n.d. & n.d. & n.d. & n.d. \\
\hline & 17 & n.d. & n.d. & n.d. & n.d. & n.d. & n.d. \\
\hline & 28 & n.d. & n.d. & n.d. & n.d. & n.d. & n.d. \\
\hline & 59 & n.d. & n.d. & n.d. & n.d. & n.d. & n.d. \\
\hline & 94 & n.d. & n.d. & n.d. & n.d. & n.d. & n.d. \\
\hline & 129 & n.d. & n.d. & n.d. & n.d. & n.d. & n.d. \\
\hline & 164 & n.d. & n.d. & n.d. & n.d. & n.d. & n.d. \\
\hline
\end{tabular}

n.d. = not detected . 
(a)

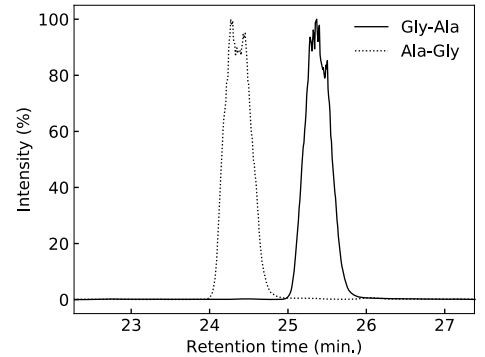

(d)

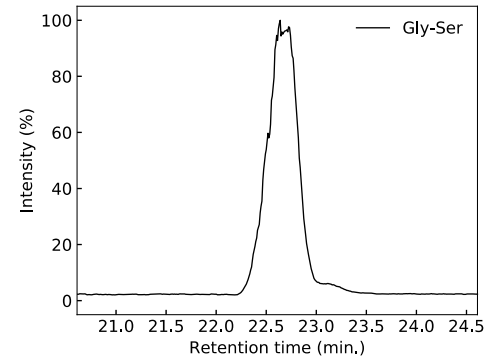

(g)

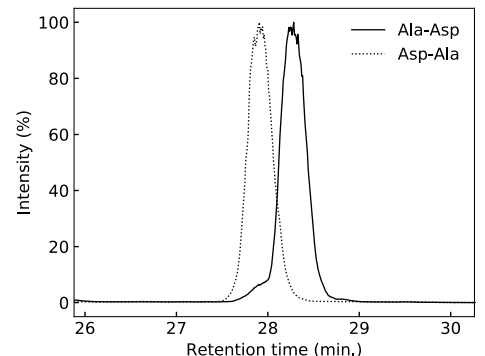

(j)

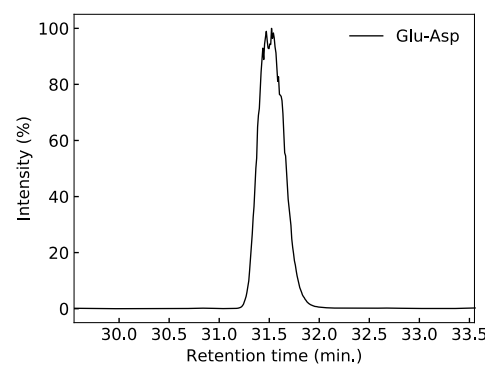

(m)

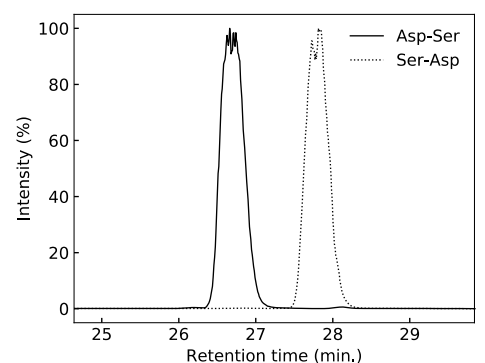

(b)

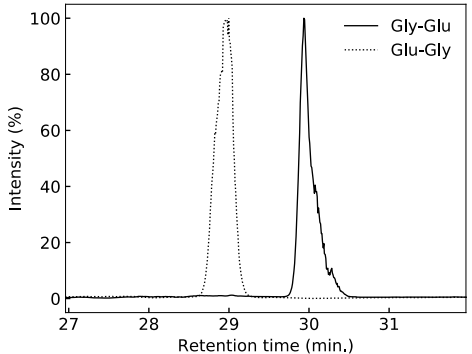

(e)

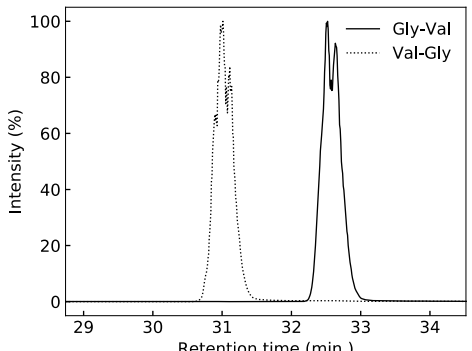

(h)

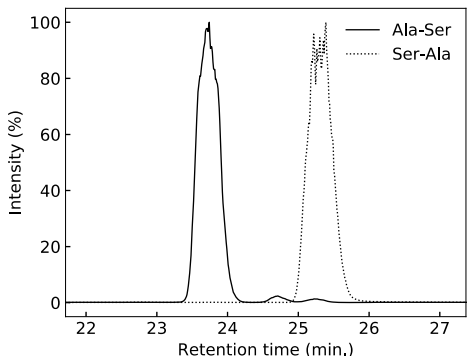

(k)

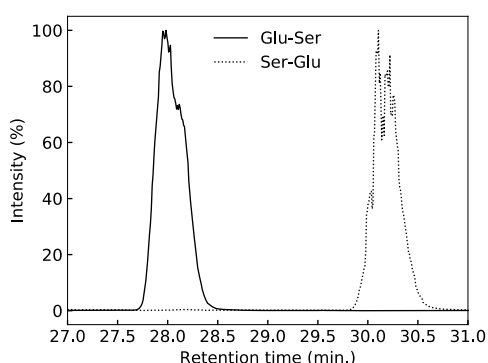

(n)

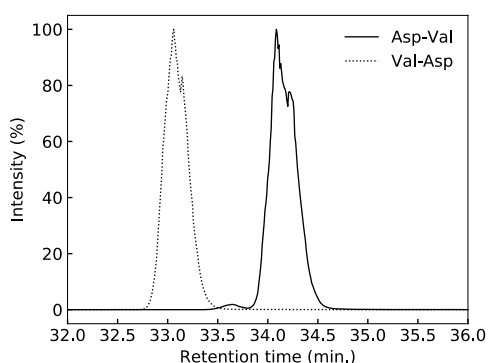

(c)

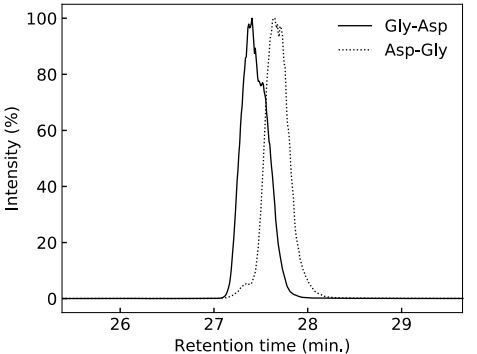

(f)

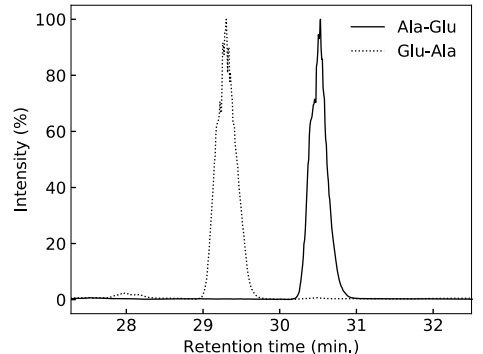

(i)

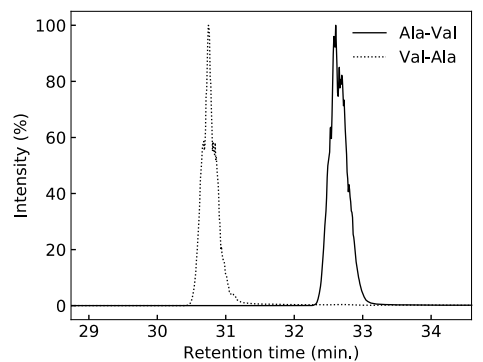

(

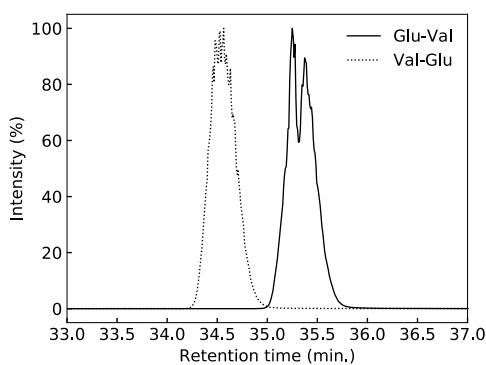

(o)

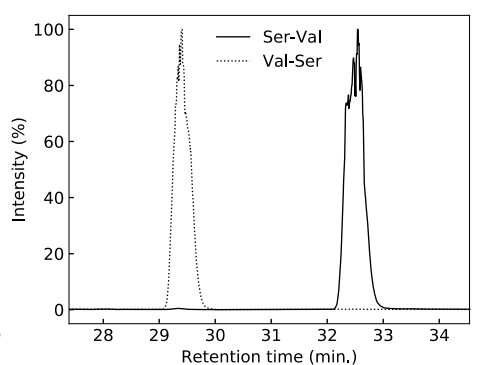

Figure S1. Representative single mass chromatogram of dipeptides. 
Dipeptides consist of (a) Gly and Ala (Mass = 146.069); (b) Gly and Glu (Mass = 204.075); (c) Gly and Asp (Mass = 190.059); (d) Gly-Ser (Mass = 162.064); (e) Gly and Val (Mass = 174.100); (f) Ala and Glu (Mass = 218.090); (g) Ala and Asp (Mass = 204.075); (h) Ala and Ser (Mass = 176.080); (i) Ala and Val (Mass = 188.116); (j) Glu-Asp (Mass = 262.080); (k) Glu and Ser (Mass = 234.085); (1) Glu and Val (Mass = 246.122); (m) Asp and Ser (Mass =220.070); (n) Asp and Val (Mass=232.106); (o) Ser and Val (Mass $=204.111)$.

Ala- dipeptide concentration $(\mu \mathrm{M})$
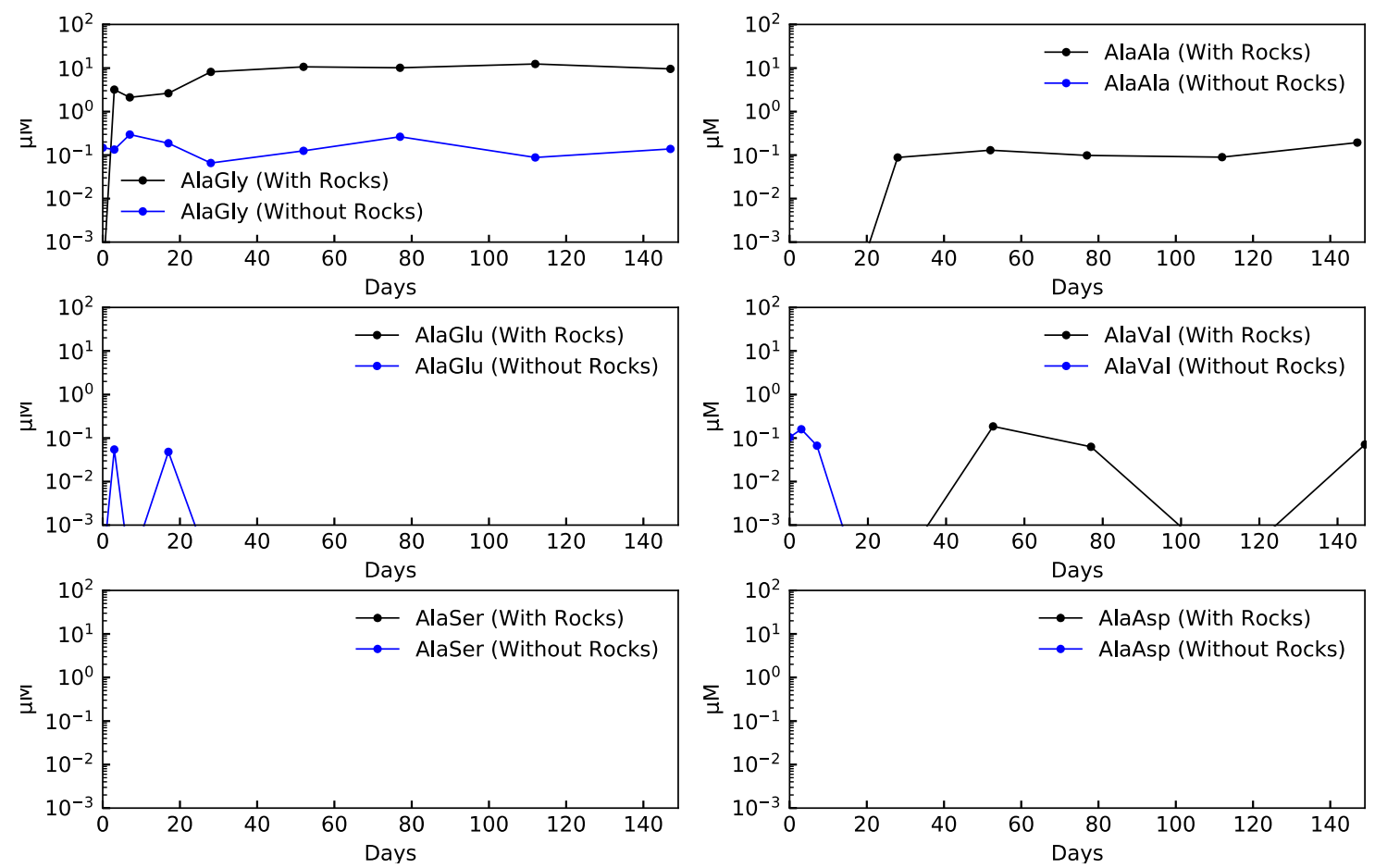

Figure S2a. Time series patterns showing the formation of dipeptides. 
Asp- dipeptide concentration $(\mu \mathrm{M})$
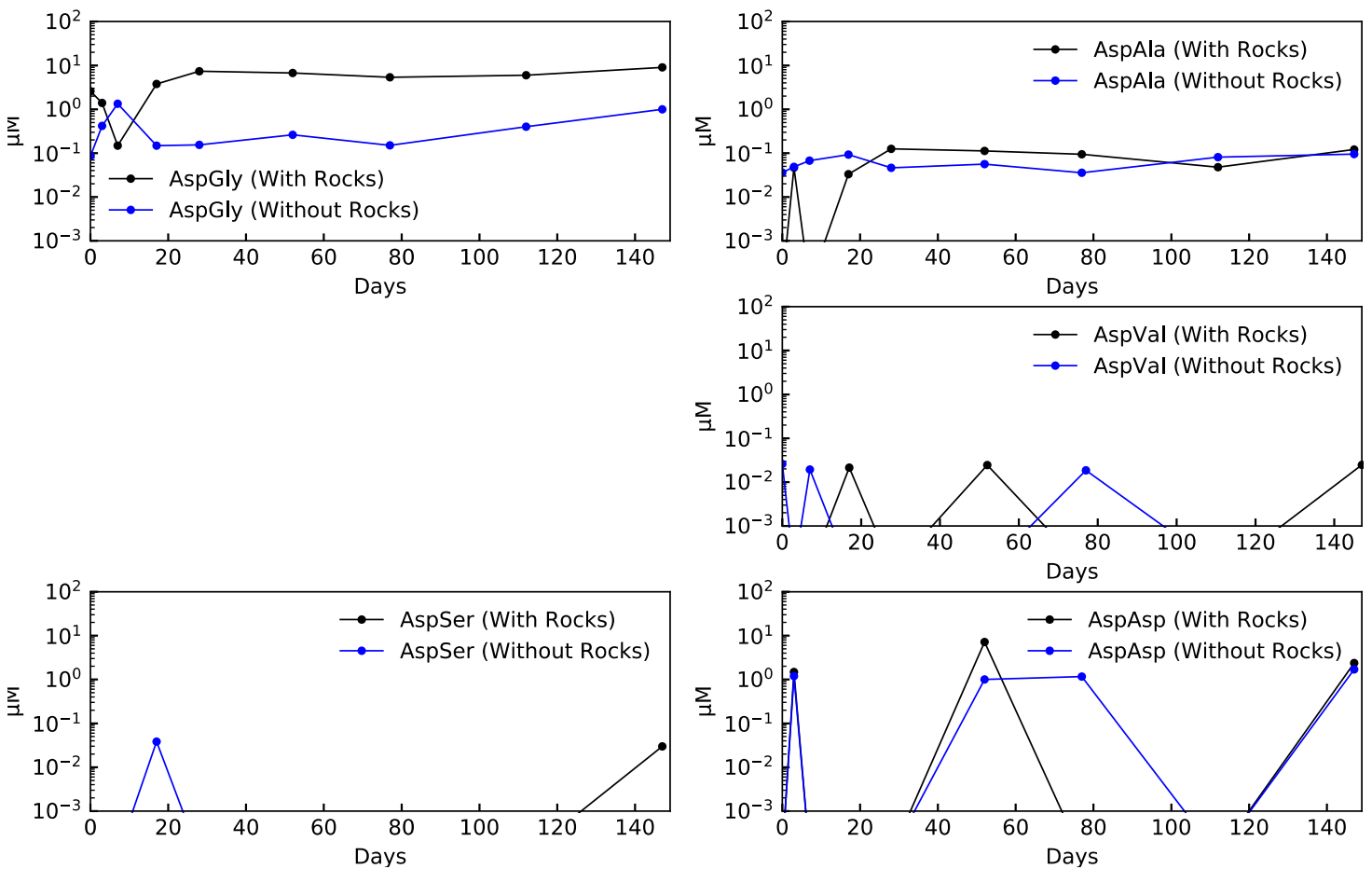

Figure S2b. Time series patterns showing the formation of dipeptides.

Glu- dipeptide concentration ( $\mu \mathrm{M})$
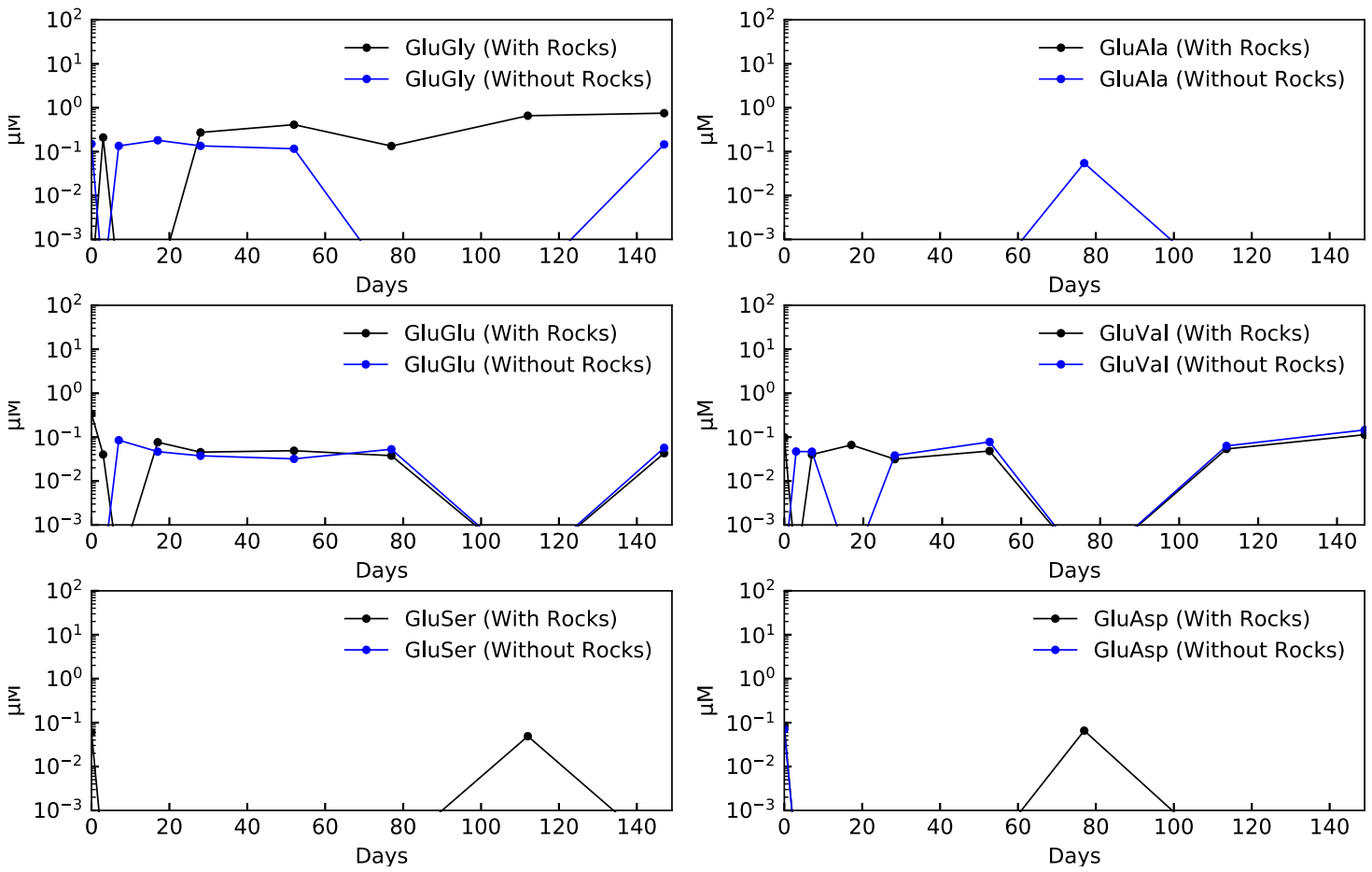

Figure S2c. Time series patterns showing the formation of dipeptides. 
Gly- dipeptide concentration $(\mu \mathrm{M})$
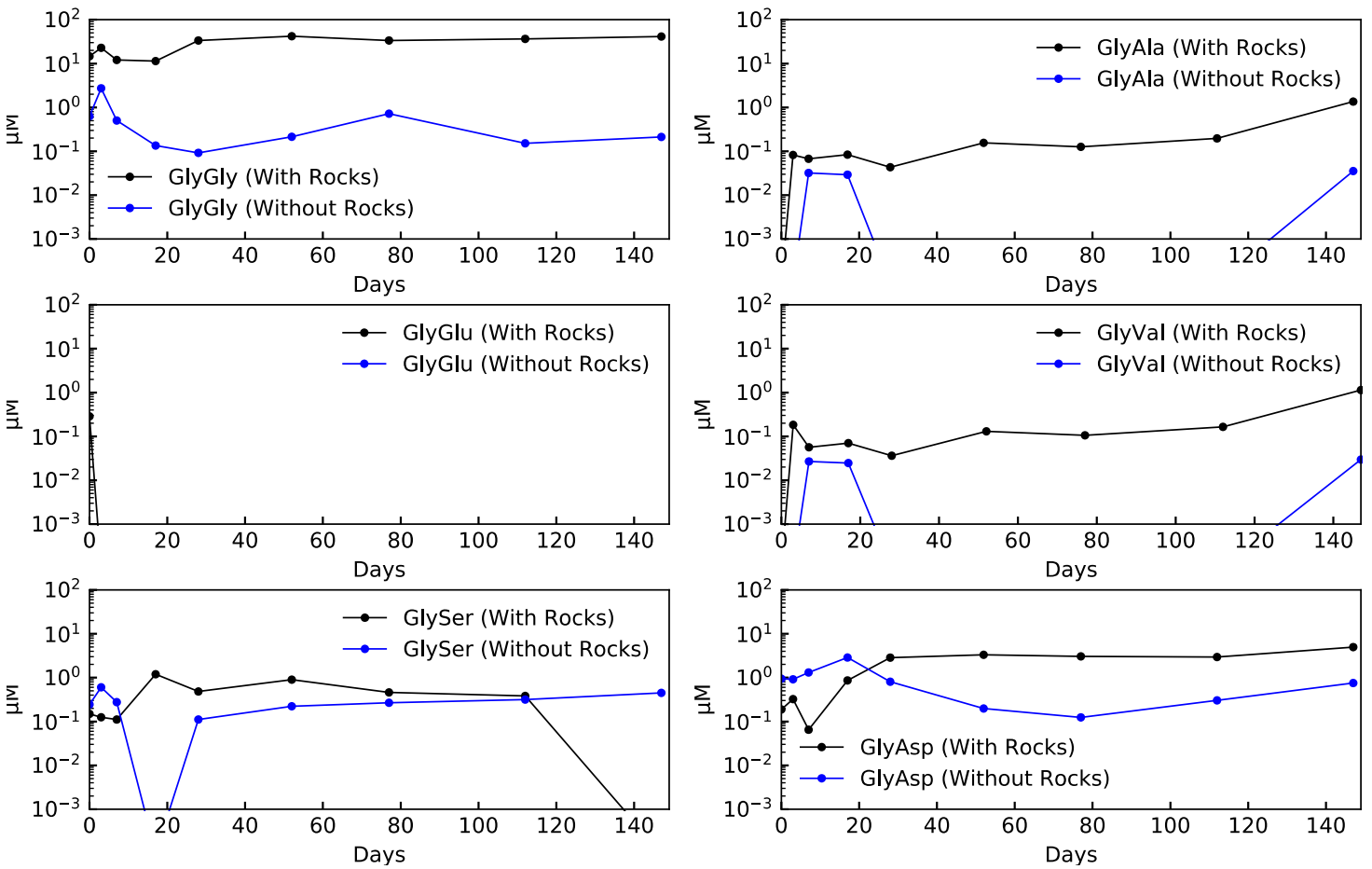

Figure S2d. Time series patterns showing the formation of dipeptides.

Ser- dipeptide concentration $(\mu \mathrm{M})$
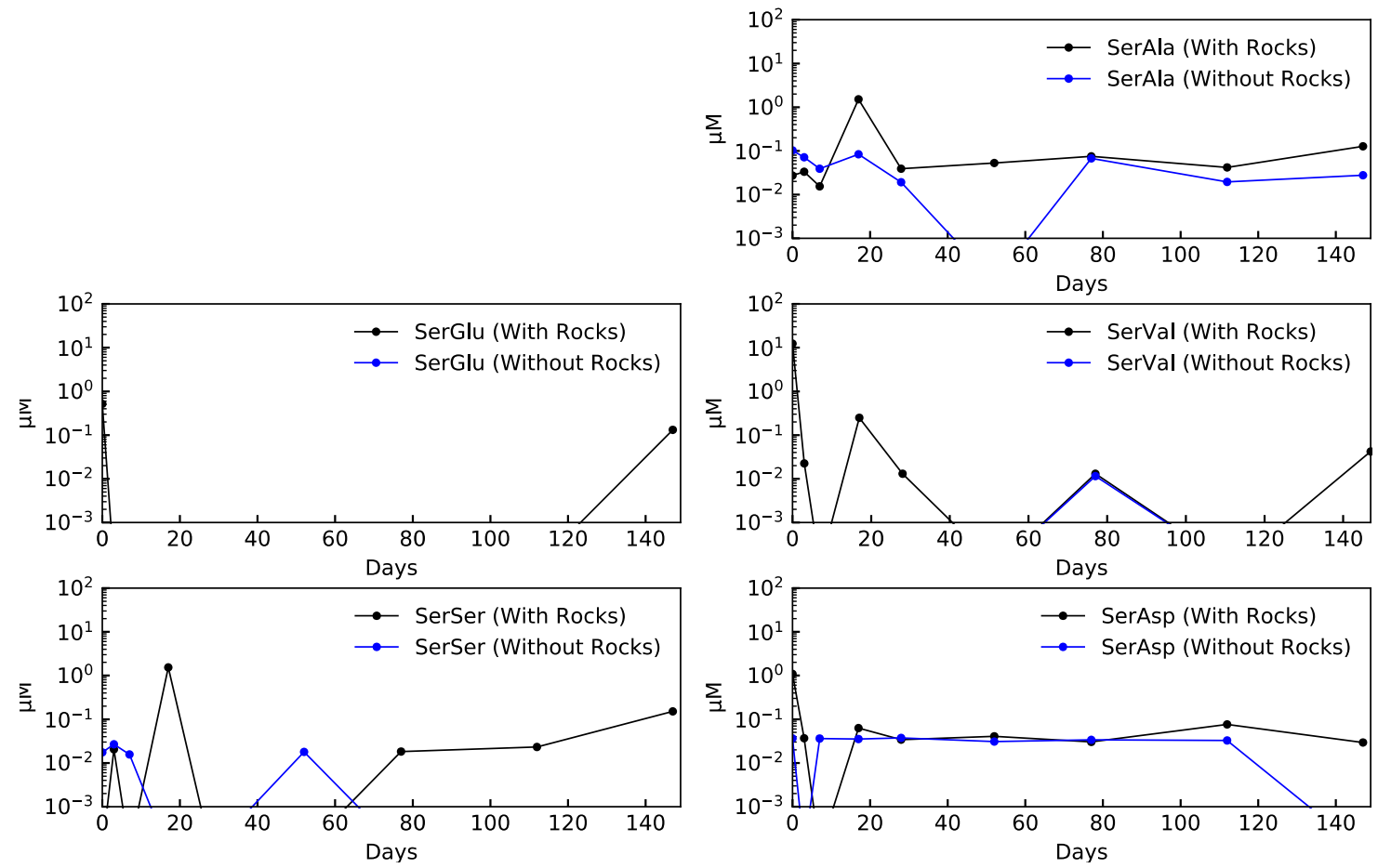

Figure S2e. Time series patterns showing the formation of dipeptides. 
Manuscript for ACS Earth and Space Chem.

Supplementary Information

Val- dipeptide concentration $(\mu \mathrm{M})$
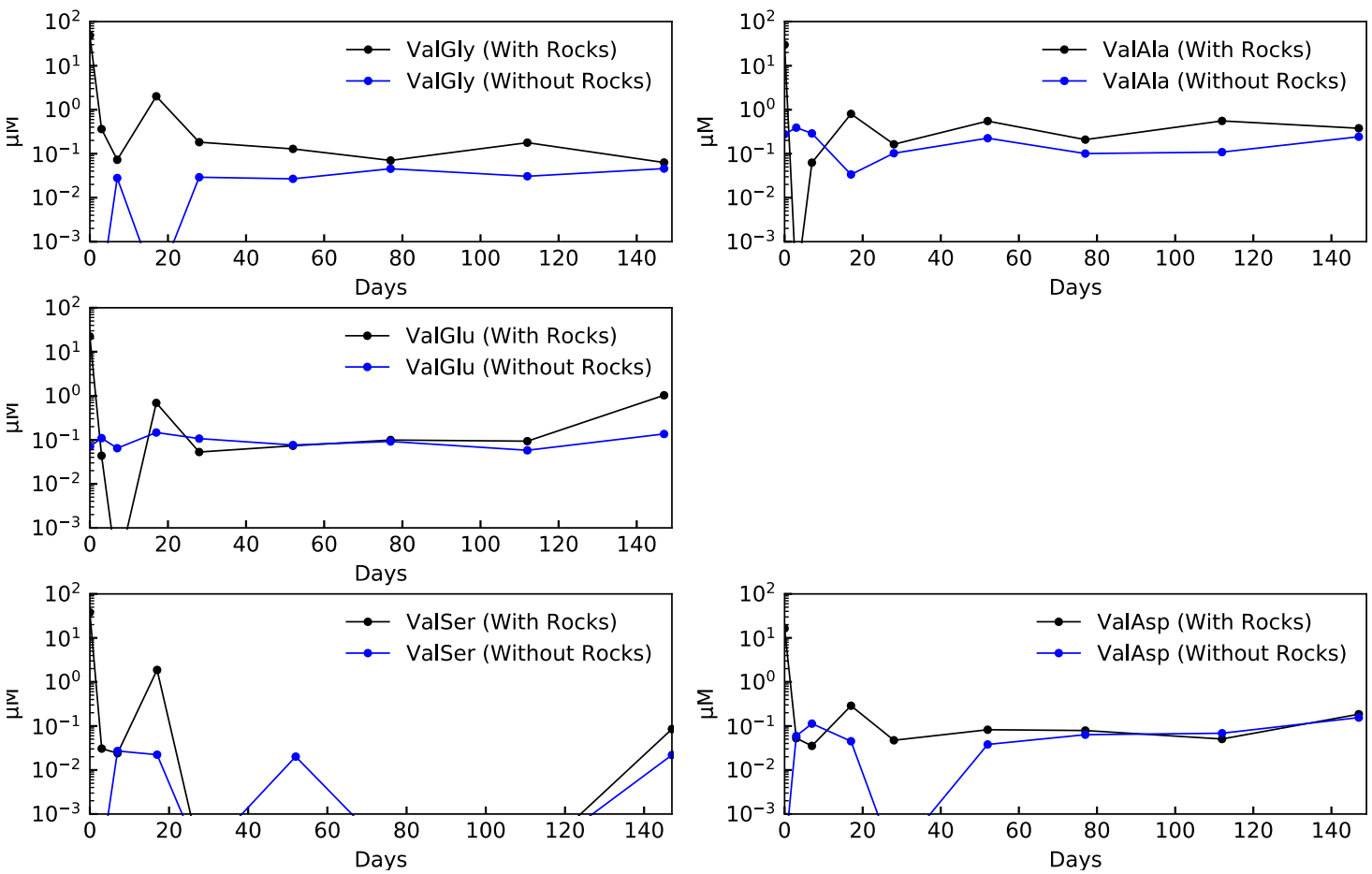

Figure S2f. Time series patterns showing the formation of dipeptides. 